\title{
Paniculite Mesentérica (PM) e Fibromatose Mesentérica (FM) - Relato de Casos
}

\author{
Mesenteric Panniculitis (MP) and Mesenteric Fibromatosis (MF) - \\ Report of Cases
}

\author{
ANTÔNIO BALESTRIM FILHO ${ }^{1}$; ROBERTALAGES DAS MERCÊS'; PAULO HENRIQUE PISI'; \\ THIAGO ANTONIO BRAGA ${ }^{3}$; ANA LUIZANORMANHAR. ALMEIDA ${ }^{4} ;$ ROGÉRIO SERAFIM PARRA $^{4}$; \\ OMAR FERES5; JOSÉ JOAQUIM RIBEIRODA ROCHA ${ }^{5}$
}

\begin{abstract}
${ }^{1}$ Médico Contratado da Fundação de Apoio, Ensino e Pesquisa - FAEPA, junto ao Hospital Estadual de Ribeirão Preto;
${ }^{2}$ Ex-Residente da Divisão de Coloproctologia do Departamento de Cirurgia e Anatomia da FMRP-USP; ${ }^{3}$ Médico Residente da Divisão de Coloproctologia do Departamento de Cirurgia e Anatomia da FMRP-USP; ${ }^{4}$ Médico Assistente da Divisão de Coloproctologia do Departamento de Cirurgia e Anatomia da FMRP-USP; ${ }^{5}$ Professor Doutor da Divisão de Coloproctologia do Departamento de Cirurgia e Anatomia da FMRP-USP.
\end{abstract}

\begin{abstract}
BALESTRIM FILHO A; MERCÊS RL; PISI PH; BRAGA TAALMEIDA ALNR; PARRA RS; FERES O; ROCHA JJR. Paniculite Mesentérica (PM) e Fibromatose Mesentérica (FM) - Relato de Casos. Rev bras Coloproct, 2009;29(3): 389-392.

RESUMO: A paniculite mesentérica (PM) e a fibromatose mesentérica (FM) são doenças fibróticas de etiologia incerta. São mais frequentes no mesentério do intestino delgado, no grande omento e nos mesocólons. Clinicamente a FM pode apresentar-se de forma aguda na qual uma complicação da doença é sua primeira expressão. Na forma crônica é caracterizada por sintomas abdominais vagos e/ou massa abdominal palpável. A comprovação diagnóstica, assim como na PM, é feita através de laparotomia exploradora ou videolaparoscopia diagnóstica e biopsia. Os autores relatam dois casos sendo um de PM e outro de FM, apresentam um paralelo dos aspectos clínicos, tomográficos, diagnóstico, histopatológico e terapêutico destacando as semelhanças e as diferenças entre essas duas patologias.
\end{abstract}

Descritores: Paniculite mesentérica; Fibrose mesentérica; Cirurgia.

\section{INTRODUÇÃO}

A paniculite mesentérica (PM) é uma doença fibrótica caracterizada por processo inflamatório crônico do tecido adiposo do mesentério ${ }^{1-7}$. Clinicamente os achados estão relacionados à inflamação e ao efeito de massa. A fibromatose mesentérica (FM) também é uma doença fibrótica de etiologia desconheci$\mathrm{da}^{13}$ e caracteriza-se por achado histopatológico de proliferação fibroblástica com deposição de colágeno no tecido mesentérico ${ }^{8-14}$, porém sem evidências de processo inflamatório ${ }^{9-11}$ ou atipias celulares que sugiram neoplasia ${ }^{8-14}$. A FM tem caráter localmente infiltrativo invadindo as alças adjacentes ${ }^{8-11,13,14}$.

\section{OBJETIVO}

Relatar de 2 casos, sendo um de PM e outro de FM e apresentar um paralelo dos aspectos clínicos, tomográficos, diagnóstico, histopatológico e terapêutico, destacando as semelhanças e as diferenças entre essas duas patologias.

\section{RELATO DE CASOS}

$1^{o}$ caso - HRD, 37 anos, masculino, comerciante com queixa há 3 anos dor abdominal tipo ardor em hipocôndrio esquerdo e irradiação para mesogástrio de caráter intermitente associado à sensação de

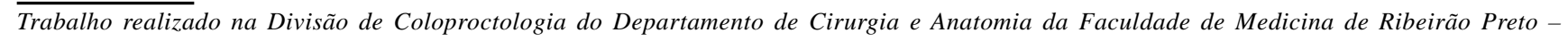
Universidade de São Paulo. 
distensão abdominal. Não relaciona com alimentação ou hábito intestinal que é normal. Nega emagrecimento. Fez uso de várias medicações para síndrome do cólon irritável sem resposta. Antecedentes: colecistectomia e apendicectomia videolaparoscópica há 2,5 anos em razão da dor, porém sem melhora. Durante esse período fez tratamento psiquiátrico com uso de várias medicações também sem melhora. Ao exame físico apresentava-se em bom estado geral, nutrido, corado, anictérico, sem alterações cardiorrespiratórias, abdome simétrico, flácido, com dor a palpação profunda hipocôndrio esquerdo e mesogástrio, sem massas palpáveis. Exames subsidiários: enema opaco com divertículos no cólon direito, trânsito intestinal e tomografia de abdome, normais. Indicado laparotomia exploradora devido a dor abdominal a esclarecer. $1^{a}$ operação em jan./06: achado múltiplos nódulos firmes variando de 3-6 cm de diâmetro na gordura do mesentério junto ao jejuno e ângulo de Treitz. Foram excisados alguns para análise histopatológica que revelou PM (Figura 1).

O paciente evoluiu com obstrução intestinal, sendo necessárias três novas abordagens abdominais. Internação longa necessitando de nutrição parenteral e antibióticos. Foram utilizados corticoterapia prolongada e colchicina com melhora gradativa. Após 115 dias de internação obteve alta e no seguimento ambulatorial apresenta ganho de peso, menos dor, hábito intestinal regular e em retirada de corticoides. $2^{o}$ caso - CFG, 43 anos, masculino, agricultor, com queixa de dor abdominal difusa inespecífica mais intensa em fossa ilíaca direita há vários meses. Hábito intestinal sem alterações. Há 10 dias com piora dos sintomas e dor persistente. Indicado laparotomia exploradora com hipótese diagnóstica de abdome agudo inflamatório (apendicite?). Achado cirúrgico de espessamento do mesentério do íleo terminal. Realizado biópsia cujo anatomo-patológico sugeria FM (Figura 2).

Evoluiu com obstrução intestinal e foi reoperado no $10^{\circ}$ dia após a $1^{\mathrm{a}}$. cirurgia. Achado: bloco de alças firmemente aderidas com fibrose do meso envolvendo íleo terminal apêndice e ceco, espessamento do meso desde a alça até retroperitônio. Feito ressecção do íleo $(60 \mathrm{~cm})$ e ceco em bloco com a fibrose do mesentério, ileostomia terminal e sepultamento do cólon ascendente. Anatomo-patológico: FM. Boa evolução pós-operatória, reconstituído trânsito intestinal. Em seguimento ambulatorial sem queixas, sem medicações.

\section{DISCUSSÃO}

PM e FM são duas doenças fibróticas distintas de etiologia desconhecida e características histológicas próprias, porém com expressões clínicas muito parecidas. Do ponto de vista histopatológico, há 3 estágios na progressão da doença $a^{1,3,4,6,7}:$ o $1^{\circ}$ estágio é marcado por degeneração gordurosa (lipodistrofia mesentérica), o $2^{\circ}$ por reação inflamatória (paniculite mesentérica) e o $3^{\circ}$ é caracterizado por fibrose (mesenterite retrátil) $)^{1,2,4,6,7}$. A etiologia é incerta ${ }^{1-7}$ mas sugere-se que componente auto-imune, coexistência com neoplasias malignas (como linfomas) e presença de trauma e cirurgia abdominais prévias estão associa$\operatorname{dos}^{1-3,6,7}$. Os homens são duas vezes mais afetados que

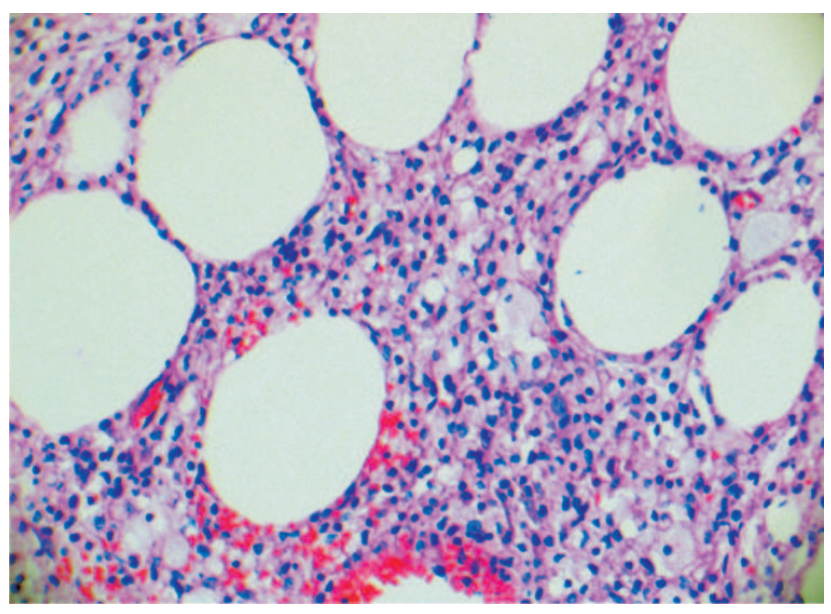

Figura 1 - Lâmina corada com hematoxilina e eosina evidenciando tecido adiposo mesenterial com grande infiltrado inflamatório presente na paniculite mesentérica.

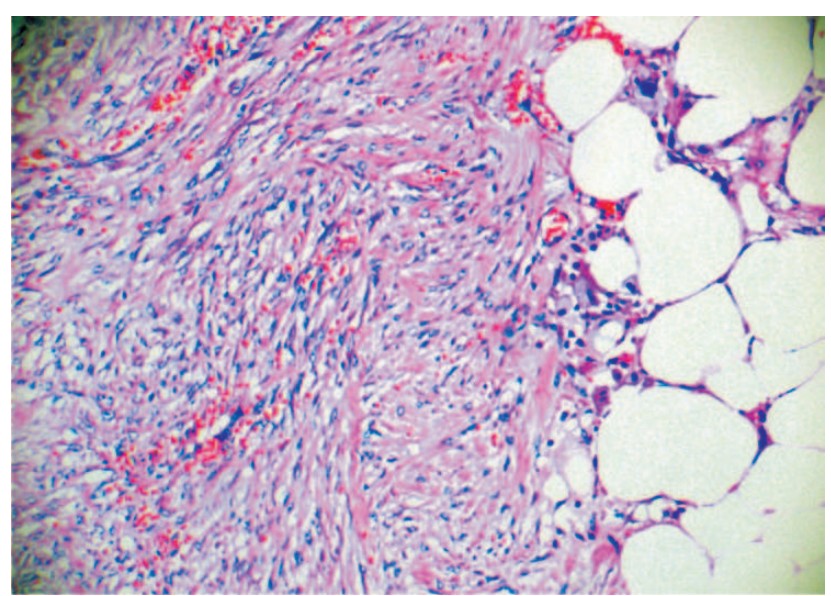

Figura 2 - Lâmina corada com hematoxilina e eosina mostrando fibroblasto e depósitos de colágeno na gordura mesenterial característico de fibromatose mesentérica. 
as mulheres ${ }^{2,4}$ e a faixa etária de pico da doença é entre 40 e $60 \operatorname{anos}^{2,4}$. O diagnóstico é confirmado através da histologia das lesões ${ }^{1-7}$ mas pode ser presumido por exames de imagem como tomografia ${ }^{1-4,6,7}$ e ressonância ${ }^{2}$. Na tomografia computadorizada existem detalhes que sugerem o diagnóstico de $\mathrm{PM}^{1-4,6,7}$. No entanto, o diagnóstico é estabelecido por laparotomia exploradora (LE) ou videolaparoscopia diagnóstica e biópsia $^{1-7}$. A fibrose mesentérica pode estar associada à síndrome de Gardner (polipose familiar, osteomas e cistos desmóides) ${ }^{8-14}$ e também a cirurgias ou traumas abdominais prévios ${ }^{9-11,13}$. A localização das lesões é mais frequente no mesentério, seguindo-se o grande omento e o mesocólon ${ }^{9-11}$. Clinicamente a FM pode apresentar-se de forma aguda na qual a obstrução é sua primeira expressão ${ }^{11,12}$. A forma crônica é caracterizada por sintomas abdominais vagos e/ou massa abdominal palpável $^{8,11-13}$. Na investigação diagnóstica a tomografia é inespecífica ${ }^{9-11,13,14}$. A comprovação diagnóstica, assim como na PM, é feita através de laparotomia exploradora ou videolaparoscopia diagnóstica e biópsia9-11,14.

A terapêutica da PM utiliza corticosteroides, imunossupressores e colchicina nos casos sintomáticos sem complicações ${ }^{1-4,6,7}$. O tratamento cirúrgico está indicado nas complicações como obstrução e/ou isquemia intestinal $^{1-4,6,7}$. Na FM a ressecção cirúrgica completa das lesões é consenso, quando possível ${ }^{8,10,11,13,14}$. Entretanto, nos casos irresecáveis, a derivação intestinal pode ser a única alternativa $8,10,11,13,14$. Tamoxifeno e anti-inflamatórios não esteroidais têm sido empregados como adjuvantes ${ }^{12,14}$ com resultados insatisfatórios $10,11,13$.

\begin{abstract}
Mesenteric panniculitis (MP) and mesenteric fibromatosis (MF) are fibrotic diseases of uncertain aetiology. Both occur most frequently in the mesentery of the small intestine, the greater omentum and the mesocolons. In its acute form, the first clinical symptoms of MF are complications of the disease. Chronic MF is characterized by vague abdominal symptoms and/ or a palpable abdominal mass. Like mesenteric panniculitis, mesenteric fibromatosis can be diagnostically verified by exploratory laparotomy, or diagnostic video-laparoscopy and biopsy. Here, we present two cases, one of MP and the other of MF, with resembling clinical and tomographic aspects, as well as similarities in diagnosis, histopathology and therapy. With our case report, we want to emphasise both similarities and differences between these two pathologies.
\end{abstract}

Key words: Mesenteric panniculitis, mesenteric fibromatosis, surgery.

\section{REFERÊNCIAS}

1. Cabral, MATH. Paniculite mesentérica -Relato de Caso. http/ /www.cbr.org.br/ cbradiologia2005/paineis/01/197/197

2. Moreira LBM, Pineiro RA, Melo ASA, Alves JRD, Noro F, Marchiori E. Paniculite mesentérica: aspectos na tomografia computadorizada. Radiol Bras. 2001;34(3): 135-140

3. Piessen G, Mariette C. Triboulet JP. Mesenteric paniculitis. Ann Chir. 2006 Feb; 131(2):85-9

4. Mitsuru SEO, Mitsuo O, Okina S, Ohdera K. Nakashima R. Sakisaka S 1. Mesenteric panniculitis of the colon with obstruction of the inferior mesenteric vein: report of a case. Dis Colon Rectum. 2001 Jun; 44(6):885-9

5. Oomori, S.. Gastrointestinal: Mesenteric panniculitis of the transverse colon.Journal of Gastroenterology and Hepatology 2007;22:596.

6. Caron II, Savoye G, Legros JR, Collet CS, Herve S, Lerebours E. Successful management of symptoms of steroid-dependent mesenteric panniculitis with colchicine.Digestive diseases and sciences. 2006 jul;51(7):1245-9.
7. Miyake H, Sano T. Kamiya J, Nagino M, Uesaka K, Yuasa N, Oda K, Nimura Y. Successful steroid therapy for postoperative mesenteric panniculitis. Surgery 2003; 133(1):118-9.

8. Holubar S, Dwivedi AJ, O’Connor J. Giant mesenteric fibromatosis presenting as small bowel obstruction. Am Surg. 2006 May; 723(5):427-9.

9. Forte MD, Brant WE. Spontaneous isollated mesenteric fibromatosis. Dis Colon Rectum. 1988 Apr; 31(4):315-7.

10. Jaadan SAA, Rabeeah AL. Mesenteric fibromatosis: case report and literature review. J Pediat Surg. 1999 Jul; 34:1130-2.

11. Saad RC, Silva JH, Donoso AC, Tuder RM, Baraviera AC. Fibromatose mesentérica com invasão do cólon. Rev Bras Colo-Proct. 1990; 1(3):102-5.

12. Teik-ying NG, Mei-Due Yang, Yung FC, Chang CH. Resolution of hydronephrosis due to massive mesenteric fibromatosis using cyclo-oxygenase 2 inhibitors. Urology 2007; 70(3):591e3-e4.

13. Rampone B, Pedrazzani C, Marreli D, Pinto E. Roviello F. Updates on abdominal desmoid tumors. World J Gastroentrol. 2007 Dec 7;13(45):5985-8. 
14. Tanaka K, Yoshikawa R, Yanagi H, Gega M, Fujiwara Y, Hashimoto TT, Hirota S, Tsujimura T, Tomita N. Regression of sporadic intra-abdominal desmoid tumour following administration of non-steroidal anti-inflammatory drug. World J Surg Oncol. 2008 Feb 8;6:17.

\section{Endereço para correspondência:}

OMAR FERES

Divisão de Coloproctologia do Depto. de Cirurgia e Anatomia da FMRP-USP

Hospital das Clínicas da FMRP-USP - Campus Universitário Ribeirão Preto, SP

CEP: 14048-900

E-mail: sbcp@ sbcp.org.br 